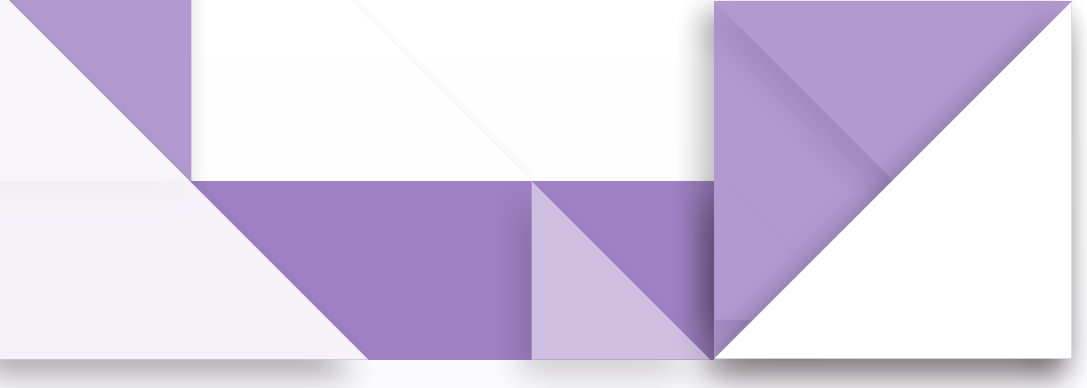

\title{
Exploración de las actitudes hacia las matemáticas de futuros profesores de educación especial
}

\section{- Exploration of Attitudes Towards Mathematics of Future Special Education Teachers}

- Exploração de atitudes frente à matemática de futuros professores de educação especial

\section{Resumen}

A partir del fenómeno de la desprofesionalización docente que predomina en la Educación Especial en México, el presente artículo de investigación tiene como objetivo explorar las actitudes hacia las matemáticas que manifiestan futuros profesores de esta modalidad del sistema educativo mexicano, ante la competencia profesional que declara el asesoramiento frente a las dificultades de aprendizaje de esta ciencia. El estudio de las actitudes de los estudiantes podría ser un preludio para señalar el tipo de conocimiento matemático a desarrollar en los futuros profesores de Educación Especial. Para lo anterior, se aplicó una escala tipo Likert a 200 estudiantes de la Licenciatura en Educación Especial de una universidad pública en México. Los resultados (confiables bajo un análisis de consistencia interna o coeficiente alfa de Cronbach, $\alpha=0.85$ ), sugieren una tendencia positiva hacia los tres componentes característicos para la actitud: cognitivo, conductual y afectivo. En particular, el componente que presenta una mayor actitud positiva es el cognitivo, lo que evidencia que los estudiantes otorgan importancia a las matemáticas y al conocimiento de la disciplina en su formación profesional.

Palabras clave:

actitud; educación especial; educación matemática

\section{J. Marcos López-Mojica* Jaime I. García-García** José Carlos Ramírez Cruz*** Elizabeth H. Arredondo****}

\footnotetext{
* Doctor en Ciencias, especialidad Matemática Educativa; profesor invitado de la Universidad Autónoma de Guerrero, Acapulco, Guerrero, México. Correo electrónico: mojicajm@uagro.mx. Código Orcid: 0000-0002-7330-9979

** Doctor en Ciencias, especialidad Matemática Educativa; catedrático de la Universidad de Los Lagos, Osorno, Chile. Correo electrónico: jaime.garcia@ ulagos.cl. Código Orcid: 0000-0002-8799-5981

*** Maestro en Educación; catedrático de la Universidad de Guadalajara, Guadalajara, Jalisco, México. Correo electrónico: jose ramirez29@ucol.mx. Código Orcid: 0000-0002-1224-4382

**** Doctora en Ciencias, especialidad Matemática Educativa; catedrática de la Universidad de Los Lagos, Osorno, Chile. Correo electrónico: elizabeth. hernandez@ulagos.cl. Código Orcid: 0000-00025285-1603
} 


\begin{abstract}
Based on the phenomenon of teacher de-professionalization that predominates in Special Education in Mexico, the present research aimed to explore the attitudes manifested by future teachers of this modality of the Mexican educational system towards mathematics, in the face of the professional competence declared by counseling against the learning difficulties of this science. The study of attitudes could be a prelude to indicate the type of mathematical knowledge to be developed in future Special Education teachers. For the above, a Likert-type scale was applied to 200 students of the Bachelor's Degree in Special Education from a public university in Mexico. The results (reliable under an internal consistency analysis or Cronbach's alpha coefficient, $\alpha=0.85$ ) suggest a positive trend towards the three characteristic components for attitude: cognitive, behavioral, and affective. In particular, the component that presents a greater positive attitude is the cognitive one, which shows that students attach importance to mathematics and the knowledge of the discipline in their professional training.
\end{abstract}

Key words:

attitude; special education; mathematics education

\title{
Resumo
}

Com base no fenômeno da desprofissionalização docente que é dominante na Educação Especial no México, esta pesquisa teve como objetivo explorar as atitudes em relação à matemática manifestadas por futuros professores desta modalidade do sistema educacional mexicano, em face da competência profissional que declara o aconselhamento contra dificuldades de aprendizagem nesta ciência. $\bigcirc$ estudo das atitudes pode ser um prelúdio para indicar o tipo de conhecimento matemático a desenvolver nos futuros professores da Educação Especial. Para o exposto, uma escala do tipo Likert foi aplicada a 200 alunos da Licenciatura em Educação Especial de uma universidade pública do México. Os resultados (confiáveis sob uma análise de consistência interna ou coeficiente alfa de Cronbach, $\alpha=0,85$ ) sugerem uma tendência positiva para os três componentes característicos da atitude: cognitivo, comportamental e afetivo. Em específico, o componente com maior atitude positiva é o cognitivo, o que mostra que os alunos atribuem importância à matemática e ao conhecimento da disciplina na sua formação profissional.

Palavras-chave:

atitude; educação especial; educação matemática 


\section{Introducción}

A casi 25 años de la Declaración de Salamanca (Unesco, 1994) en donde 92 países, incluyendo México, se comprometieron a garantizar el acceso a la educación de manera integral a los alumnos que presentan necesidades educativas especiales (NEE) y atender a la diversidad, surge de manera natural la interrogante: ¿̇qué se ha logrado al respecto? En México surgió el Proyecto Nacional de Integración Educativa (García-Cedillo y Romero, 2019) que permitió la formalidad del proceso de integración educativa en varias esferas del sistema educativo nacional y promovió la investigación, prácticas pedagógicas innovadoras y la difusión de experiencias educativas exitosas. En la actualidad, sigue vigente en la práctica la integración educativa y de manera teórica, pero no clara, el enfoque de la educación inclusiva (García-Cedillo, 2018) en la propuesta institucional de la educación básica.

Una de las preocupaciones de la Organización de las Naciones Unidas para la Educación, la Ciencia y la Cultura (Unesco) es la formación inicial a los profesores (Unesco, 1994), pues señala que se les debe preparar en la adaptación del contenido de los programas de estudio referido a los conocimientos disciplinares; tal es el caso del área de las matemáticas. Es decir, los futuros profesores de Educación Especial deben desarrollar la competencia que permita el asesoramiento a profesores de educación regular, padres de familia y alumnos con discapacidad, frente a las dificultades de aprendizaje en matemáticas (Universidad de Colima, 2011).

En concordancia con la Declaración de Salamanca, los profesores deben desarrollar la "capacidad de evaluar las necesidades especiales, de adaptar el contenido del programa de estudios, de recurrir a la ayuda de la tecnología, de individualizar los procedimien- tos pedagógicos para responder a un mayor número de aptitudes, etc." (Principio 41 , p. 27 citado en García-Cedillo y Romero, 2019).

La preparación en la atención a la diversidad no solo compete a los profesores de Educación Especial. Al respecto, Perrenoud (2010) plantea competencias deseables en la formación de profesores a nivel básico; en particular, una de ellas propone desarrollar la capacidad de elaborar y hacer evolucionar dispositivos de diferenciación. En otras palabras, se pretende que el profesor cuente con habilidades y conocimientos didácticos que incluyen dimensiones afectivas, cognitivas y relacionales para trabajar con alumnos con dificultades de aprendizaje. El modelo de formación se fundamenta en la confianza de las capacidades de los profesores en formación para afrontar el conocimiento profesional especializado; sin embargo, la práctica marca nuevos retos a los profesores, tanto en formación como en servicio, y a las instituciones de las que egresaron para mantenerse actualizados a las necesidades emergentes (Zapata, 2019).

Así, ante el cambio de paradigma se presenta un fenómeno, 'la desprofesionalización', que puede entenderse como una serie de sentimientos de incapacidad sobre las competencias adquiridas para la función docente. En palabras de Guajardo, "la desprofesionalización es el sentimiento de despojo de las competencias adquiridas, que quedan obsoletas y resultan inútiles ante las decisiones anacrónicas de una época" (2010, p. 112). Esto se refleja en el tránsito de la atención de niños con NEE asociadas a una discapacidad bajo un enfoque clínico a un enfoque educativo.

Lo anterior se complica cuando el conocimiento que está en juego es del área de las matemáticas para la Educación Especial.

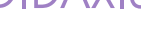


Hegarty (2008) resalta la importancia de realizar exploraciones sobre el aprendizaje de las matemáticas de niños con NEE y la formación de profesores; pues, según el autor, es una línea de investigación poco explorada en esta área del conocimiento.

Los primeros egresados de Educación Especial en México datan del año 2008 (Guajardo, 2010), bajo el enfoque de integración educativa; 14 años después de la Declaración de Salamanca. Por lo tanto, ¿̇qué se está realizando para lograr por completo la integración educativa de niños con NEE asociadas o no a una discapacidad? Romero y García-Cedillo (2013) señalan que hay resultados alentadores, pero es necesario aplicar por completo la visión de la integración educativa. Si bien aumentó la incorporación de niños con NEE (García-Cedillo y Romero, 2016) asociados a una discapacidad en las aulas de la educación regular, Flores y García-Cedillo (2016) expresan que los profesores no proporcionan los apoyos educativos a estos alumnos y dejan la atención a los profesionales de la Educación Especial en su servicio de Usaer (Unidades de Servicios de Apoyo a la Educación Regular), perteneciente a la Secretaría de Educación Pública (SEP) en México.

Dado lo anterior, la figura del profesor de Educación Especial toma relevancia, por lo que interesa indagar sobre el tipo de actitudes que presentan hacia las matemáticas según las competencias profesionales que declaran la necesidad de orientar a los estudiantes en conocimientos disciplinares. Identificar la actitud que posee el profesor, puede ser orientador respecto al conocimiento matemático que desarrollen, ya que la actitud influye en el proceso de enseñanza (Casis et ál., 2017) y en la motivación académica del estudiante (García y Pascual, 2018).

García y Pascual (2018) informan que las investigaciones en matemática educativa sobre las actitudes se han centrado en evidenciar factores de influencia en su formación, así como en crear escalas para medirlas, en modelos para explicarlas y en intervenciones para modificarlas; mismas que se han desarrollado en contextos escolares con población denominada regular. Mientras que Sánchez et ál. (2011) señalan que las actitudes de los profesores tienen influencia en sus alumnos y en su rendimiento académico. Es tanta la carga sobre estas que, actitudes como ansiedad y miedo del profesor se pueden reflejar en la conducta del estudiantado. Por lo tanto, un conocimiento adecuado de las matemáticas y de su enseñanza producirían actitudes positivas tanto en él como en sus estudiantes. Por su parte, Martínez-Padrón (2008) resalta la relación de la actitud entre lo que se aprende, lo que se enseña y lo que se evalúa, para generar ambientes de enseñanza-aprendizaje-evaluación enriquecedores y agradables, que motiven cada vez el aprendizaje de la matemática.

En consideración de lo anterior, esta investigación pretendió responder a la pregunta: ¿̇cuáles son las actitudes hacia las matemáticas que presentan los futuros profesores de educación especial? Indagar sobre sus actitudes permitirá identificar algunas características de su identidad como profesor de Educación 
Especial cuando participe en el proceso de enseñanza-aprendizaje de las matemáticas, y en la ética que posee cuando debe gestionar este tipo de aprendizaje.

\section{Antecedentes}

En México, la Educación Especial es una modalidad educativa destinada a atender a niñas, niños y jóvenes que presentan NEE asociadas o no a una discapacidad (Romero et ál., 2013) o aquellos con aptitudes sobresalientes. Por ello, se deben brindar las condiciones necesarias para que estos estudiantes puedan acceder a la formación que señala el currículo de la Educación Básica regular (SEP, 2016), particularmente el de matemáticas, en escenarios separados a las escuelas denominadas regulares.

Pero también se considera una disciplina científica (García-Cedillo y Romero, 2019) que brinda aportes a la educación en general, en relación al conocimiento de las distintas discapacidades, trastornos y aptitudes sobresalientes, encaminadas a mejores prácticas para enseñar y aprender.

Una pieza clave para ofrecer una educación de calidad es el profesor (Valdés y Monereo, 2012). Por esta razón, interesa que desde la formación inicial pueda desarrollar un conocimiento especializado y conocimiento disciplinar (Romero y García-Cedillo, 2013), con los que tengan elementos para asesorar a profesores, alumnos y padres de familia frente a las dificultades en el aprendizaje de las matemáticas de estudiantes con NEE.

En México, la formación de profesores de Educación Especial está bajo el enfoque de la integración educativa (García, 2018; Guajardo, 2010; SEP, 2004) y se caracteriza por la formación disciplinar y la práctica profesional (Guajardo, 2010). La formación disciplinar refiere a la preparación en el tratamiento educativo de los niños con discapacidad, la enseñanza de materias como español y matemáticas, además de aquellas relativas a las discapacidades. La práctica profesional es la incorporación del estudiante a un escenario real, donde aplica lo que aprendió en su formación disciplinar.

López-Mojica y Aké, (2015) justifican la existencia de dos corrientes formativas en México para profesores de Educación Especial: enfoque normalista (SEP, 2004) y enfoque universitario (Ramírez y López-Mojica, 2015). La preparación en el área de las matemáticas no difiere en ambos enfoques formativos, ya que solo cursan una asignatura relacionada con temas de aritmética, descuidando las otras áreas del conocimiento matemático, como álgebra, geometría, probabilidad y estadística, por mencionar algunas. Además, es preocupante la carencia del tratamiento de las particularidades que implicaría la enseñanza de estas áreas a personas con discapacidad, cuando los futuros profesores presentan deficiencias en su conocimiento matemático, por ejemplo, en fracciones (Ramírez et ál., 2018).

En concreto, en la formación del profesor de Educación Especial no se prevén conocimientos disciplinares específicos (Romero y García-Cedillo, 2013). Por ejemplo, el plan de formación curricular de la Licenciatura en Educación Especial de la Universidad de Colima solo ofrece un curso de matemáticas, y este no aporta a cómo enseñar y cómo adaptar los conocimientos disciplinares a las necesidades del estudiante. Además, no hay unidades de aprendizaje que incidan en el desarrollo de un pensamiento matemático del estudiante con NEE; a pesar de que los conocimientos básicos de matemáticas son elementales para una cultura general y un conocimiento para todos. 
Hasta aquí, se ha expuesto el escenario al que se enfrenta un profesor de Educación Especial, el cual es formado como un profesional de amplio espectro de competencias y que puede tener sentimientos de incapacidad cuando esas competencias deben gestionar aprendizajes. En seguida se dialogará sobre la actitud del profesor desde la investigación en educación matemática, con el fin de situar las dimensiones que recuperan las diversas investigaciones.

\section{Marco conceptual}

Una de las premisas constantes en las investigaciones sobre la actitud de los profesores en matemáticas es que, además de enseñar una serie de contenidos matemáticos, con su actitud transmiten un mensaje que influye en sus estudiantes; y una vez desarrollado esto, es difícil cambiarlo. Por ello, hay un creciente interés de investigaciones por explorar la actitud del profesor, inicialmente por un supuesto, 'las actitudes influyen en el rendimiento de los estudiantes en matemáticas', hecho que algunas investigaciones han cuestionado, pues se ha identificado que no existe una correlación directa entre el rendimiento académico y la actitud (Ma y Kishor, 1997).

Inicialmente el constructo actitud no ha tenido un consenso, pero generalmente se asocia con el agrado o desagrado con las matemáticas (García y Pascual, 2018). El propósito que se avizora con este tipo de investigaciones tiene que ver con entregar insumos para desarrollar una identidad docente que apoye a la gestión del conocimiento en matemáticas.

La actitud matemática es uno de los conceptos más complicados, ya que típicamente dentro de la investigación toma cuerpo su definición, a partir de la relación tríadica de la dimensión cognitiva (creencias), la dimensión afectiva (emociones) y la dimensión conativa (comportamiento). La dificultad de este término es su recursividad, pues a su vez define a las emociones, al comportamiento y a las creencias (Roesken et ál., 2011 ). Enseguida haremos un breve recorrido sobre la actitud en el dominio afectivo con el fin de situar nuestra investigación.

\section{La actitud en el dominio afectivo}

El estudio de los fenómenos que surgen en la enseñanza y el aprendizaje de las matemáticas ha llevado a los investigadores a explorar otras áreas del conocimiento, como la educación, la filosofía, la pedagogía y la psicología; en esta última, con el fin de comprender el proceso cognitivo que desarrollan los individuos cuando aprenden. En ese sentido, es indudable que, al tratarse de personas, el aspecto afectivo no puede ser ignorado.

Diversos acercamientos teóricos han comenzado a explicar la relación que existe entre el agrado por las matemáticas y el desempeño que este puede generar en el desarrollo del individuo y su conocimiento. Al respecto, Hannula et ál., (2004) exponen una conformación del dominio afectivo en matemática 
educativa, constituida por las emociones, creencias y actitudes; en esta investigación, es de nuestro interés lo relativo a las actitudes.

Martínez-Padrón define a la actitud como "predisposiciones comportamentales $\mathrm{u}$ orientaciones afectivas que un sujeto adquiere y que acompaña con una reacción valorativa o evaluativa manifiesta a través del agrado - el desagrado hacia algún objeto, sujeto o situación" (2008, p. 244). Asimismo, este autor considera que las actitudes son instancias que predisponen al sujeto sobre hechos de la realidad y orientan su pensamiento para adaptarlo al contexto; además de que son sentimientos positivos o negativos sobre un objeto psicológico que orienta al sujeto a actuar y expresarse.

Para Di Martino (2016), la base de la actitud es la predisposición positiva o negativa al responder ante una situación, es decir, es una predisposición con cierta carga emocional que influye en la conducta del individuo (Gil et ál., 2005). También puede considerarse como una evaluación positiva o negativa aprendida, hacia un sujeto o situación, atribuyendo un factor para su aprendizaje o su obstaculización en el aula (García y Juárez, 2011 ).

García y Juárez (2011) argumentan que las actitudes presentan tres componentes: a) afectivo, refiere a sentimientos, emociones y motivaciones; b) conductual, es la predisposición o modo de actuar; c) cognitivo, alude a pensamiento y creencias. Además, se pueden identificar tres elementos que permiten discriminar la actitud (Gil et ál., 2005): 1) creencias o pensamientos sobre el objeto de la actitud, 2) el afecto o carga evaluativa de dichas creencias, y 3) una intención de conducta con relación a la actitud.

Por su parte, Gómez-Chacón (2009) refiere la existencia de dos categorías, las actitudes hacia las matemáticas y actitudes matemáticas. La primera es la valoración al aprecio de esta disciplina, el interés por esta materia y su aprendizaje; la segunda alude a las capacidades generales para las actividades matemáticas, como la flexibilidad de pensamiento y la objetividad en el trabajo en matemáticas. En esta investigación nos enfocamos en la primera categoría.

Según Casis et ál. (2017), la actitud del profesor es un factor clave para el éxito en el aprendizaje de las matemáticas, influye en la dedicación y esfuerzo en esta materia, además de disminuir un comportamiento de rechazo hacia estas. Por ello, dependiendo del cómo se percibe una situación matemática, se tendrá un pensamiento o creencia que derive en una emoción o sentimiento y, finalmente, sobre eso es el modo de actuar de cada persona durante la clase o asesoría de matemáticas. Por lo que la actitud tiene influencia en la motivación, la perseverancia y el autoconcepto (Ruiz et ál., 2013), aspectos esenciales para el éxito escolar en matemáticas.

En esta investigación asumiremos la postura Martínez-Padrón (2008), considerando nuestro interés explorar las actitudes de los futuros profesores de Educación Especial; para ello, este autor considera los siguientes componentes en la actitud hacia las matemáticas.

- Componente cognitivo (el conocer/el saber): este componente consiste en la carga de información y experiencia adquirida por el sujeto respecto al objeto de su actitud, esta se manifiesta en sus percepciones, ideas, opiniones, concepciones y creencias.

- Componente conductual (la intención): en este componente, los sujetos manifiestan su inclinación voluntaria para realizar una acción, es decir, se constituye por predisposiciones, predilecciones, preferencias y tendencias. 
- Componente afectivo (la emoción/ el sentir): en este componente se ponen de manifiesto las emociones, los sentimientos de aceptación y rechazo, que los sujetos activan por objetos, personas o situaciones.

\section{Metodología}

Esta investigación se desprende de un proyecto que se interesó por indagar acerca del tipo de contenido matemático necesario para la formación de futuros profesores de Educación Especial. Como estado previo, se explora la actitud hacia las matemáticas que atribuyen las y los estudiantes de Licenciatura en Educación Especial de una universidad pública en México.

La investigación de tipo cuantitativa (Palella y Martins, 2012), con enfoque exploratorio (Monje, 201 1), se desarrolló en tres fases. La primera consistió en documentarse sobre la formación matemática y las actitudes hacia las matemáticas para constituir un marco de referencia. La segunda fase se dedicó a construir una escala tipo Likert sobre las actitudes hacia las matemáticas; el instrumento se sometió a jueceo (validez de contenido) por parte de expertos en didáctica de las matemáticas, psicometría y Educación Especial. La tercera fase correspondió al análisis de la aplicación de la escala y a la presentación de los resultados.

El instrumento se aplicó a 200 estudiantes de la Licenciatura en Educación Especial de la Universidad de Colima, perteneciente a la Facultad de Ciencias de la Educación, en México. De la población, 15 (7.5\%) eran hombres y 185 $(92.5 \%)$ mujeres, en su mayoría solteros. El 21 \% trabajaba, en concreto, en el sector educativo. Los participantes pertenecían a diferentes semestres de la carrera, tal como se muestra en la Tabla 1.

Tabla 1. Distribución de los estudiantes según semestre

\begin{tabular}{lccc}
\hline Semestre & Mujeres & Hombres & Total \\
\hline Segundo & 48 & 2 & 50 \\
\hline Cuarto & 55 & 4 & 59 \\
\hline Sexto & 40 & 7 & 47 \\
\hline Octavo & 42 & 2 & 44 \\
\hline Total & 185 & 15 & 200 \\
\hline
\end{tabular}

Fuente: elaboración propia.

La Licenciatura en Educación Especial se cursa en cuatro años (ocho semestres) y es modular. El único acercamiento a las matemáticas lo tienen en la unidad de aprendizaje "adquisición, alteraciones y estrategias de atención en las matemáticas" que se cursa en el quinto semestre perteneciente al módulo de Lenguaje (Universidad de Colima, 2011 1). Por lo tanto, los participantes de sexto y octavo semestre ya la habían cursado y estaban en prácticas en escenarios reales (etapa de formación Práctica Profesional). Los 200 estudiantes participaron de manera voluntaria y sin ningún tipo de recompensa. 


\section{El instrumento de la investigación}

En la segunda fase se diseñó y validó una escala de tipo Likert de 5 puntos para identificar las actitudes que presentan los estudiantes hacia las matemáticas (Gómez-Chacón, 2009). Las opciones de respuestas van de 1 (totalmente en desacuerdo) a 5 (muy de acuerdo). La escala se forma de dos partes, la primera concierne a datos sociodemográficos y escolares, además de un consentimiento informado de la investigación; la segunda parte contiene los 18 ítems, divididos en tres componentes: cognitivo, conductual y afectivo. En la Tabla 2 se muestran los ítems según el componente correspondiente.

Tabla 2. Componentes de la escala y su correspondencia con los ítems

\begin{tabular}{ll}
\hline Componente & Ítems \\
\hline Cognitivo: se evalúa la importancia, conocimiento, habilidad y dominio en matemáticas & $1+, 2+, 3+, 4-$ y $10+$ \\
\hline & \\
$\begin{array}{l}\text { Conductual: se evalúa la predisposición que se tiene hacia la asignatura de matemáticas y } \\
\text { su contenido, su relevancia en la formación profesional y en la vida cotidiana, así como la } \\
\text { actitud del profesor al impartir la clase }\end{array}$ & $5+, 6-, 7-, 8-, 12+, 14-$ y \\
& \\
\hline $\begin{array}{l}\text { Afectivo: se identifican las emociones y sentimientos que puede tener el estudiante al } \\
\text { momento de efectuar alguna actividad que implique el uso de las matemáticas; algunas } \\
\text { expresiones son: gusto hacia su estudio, sentimientos de temor, aburrimiento, dificultad o } \\
\text { tranquilidad }\end{array}$ & $9+, 11-, 13-, 15+, 16-$ y \\
\hline
\end{tabular}

Fuente: elaboración propia.

Nota: El signo $(+,-)$ indica la dirección del ítem.

La aplicación de la escala es de manera individual y dura aproximadamente 15 minutos. Consiste en colocar una " $X$ " en la casilla que corresponda a la opción con la que más se identifique el estudiante. Para analizarla se obtienen las medias en cada uno de los componentes de forma que altos puntajes denotan una actitud positiva, mientras que bajas corresponden a una actitud negativa. El instrumento (Tabla 3) se deriva del trabajo de Fennema y Sherman (1976), quienes presentaron una escala para medir actitudes en matemáticas. Por lo que, en Ramírez et ál. (2018) se justifica la construcción del instrumento en su validez y confiabilidad.

Tabla 3. Escala para medir actitudes en matemáticas

\begin{tabular}{ll}
\hline Ítem & Descripción \\
\hline 1 & $\begin{array}{l}\text { Cuando me enfrento a una actividad matemática para la educación especial me siento capaz de } \\
\text { resolverla }\end{array}$ \\
\hline 2 & $\begin{array}{l}\text { Para el desarrollo profesional de mi carrera, una de las asignaturas importantes que se estudian } \\
\text { son las matemáticas }\end{array}$ \\
\hline 3 & $\begin{array}{l}\text { Necesito un alto dominio de las matemáticas para mi futuro trabajo en personas con } \\
\text { discapacidad, alteración o aptitud sobresaliente }\end{array}$ \\
\hline 10 & Me considero poco hábil en matemáticas \\
\hline
\end{tabular}




\begin{tabular}{|c|c|c|}
\hline 5 & $\begin{array}{l}\text { Me parece completo el contenido matemático que lleva la licenciatura en } \\
\text { educación especial }\end{array}$ & \multirow{7}{*}{ Conductual } \\
\hline 6 & Las matemáticas resultan poco relevantes en mi vida & \\
\hline 7 & Considero que las matemáticas son innecesarias como asignatura & \\
\hline 8 & $\begin{array}{l}\text { Me gustaría que se omitiera la asignatura de matemáticas dentro de mi } \\
\text { carrera }\end{array}$ & \\
\hline 12 & $\begin{array}{l}\text { Considero indispensable la buena disposición del profesor al impartir la } \\
\text { asignatura de matemáticas }\end{array}$ & \\
\hline 14 & Considero poco indispensable la asignatura de matemáticas en mi carrera & \\
\hline 18 & Las matemáticas las aplico en mi vida cotidiana & \\
\hline 9 & $\begin{array}{l}\text { Me siento tranquilo cuando tengo que trabajar en actividades que impliquen } \\
\text { las matemáticas }\end{array}$ & \multirow{6}{*}{ Afectivo } \\
\hline 11 & Aunque estudie matemáticas son difíciles para mí & \\
\hline 13 & Por lo regular me estreso en un examen de matemáticas & \\
\hline 15 & Tengo gusto hacía las matemáticas & \\
\hline 16 & Las matemáticas es una de las asignaturas que más temo & \\
\hline 17 & Considero a las matemáticas difíciles y aburridas & \\
\hline
\end{tabular}

Fuente: elaboración propia.

Respecto al atributo de validez, se aplicó el juicio de expertos en el área de Matemática Educativa y Educación Especial, para evaluar su redacción y el contenido de ítems, y para reflexionar si dicho instrumento mide el constructo teórico de interés (Ramírez et ál., 2018). Para el atributo de confiabilidad, se aplicó un análisis de consistencia interna o coeficiente alfa de Cronbach, obteniendo un valor de 0.85, que implica una alta consistencia.

\section{Resultados: exploración de las actitudes}

Bajo la premisa de que las actitudes son predisposiciones con carga emocional e influyen en la conducta del individuo (Di Martino, 2016; Gil et ál., 2005) y con la acepción señalada por Gómez-Chacón (2009) sobre actitudes hacia las matemáticas, los resultados que se presentan a continuación exhiben, de manera general, las actitudes de los futuros profesores de Educación Especial.

La Tabla 4 presenta, grosso modo, las actitudes de los 200 participantes tomando como referencia las medias, y las desviaciones estándar, de las puntuaciones dadas por cada uno de ellos en los ítems según los componentes cognitivo, conductual y afectivo. 
Tabla 4. Medias y desviaciones estándar de las puntuaciones por componente e ítem en la actitud hacia las matemáticas

\begin{tabular}{|c|c|c|c|c|c|}
\hline Componente & Media & Desviación estándar & Ítem & Media & Desviación estándar \\
\hline \multirow{5}{*}{ Cognitivo } & \multirow{5}{*}{3,27} & \multirow{5}{*}{1,10} & 1 & 3,86 & 0,84 \\
\hline & & & 2 & 3,42 & 1,10 \\
\hline & & & 3 & 3,40 & 1,03 \\
\hline & & & 4 & 2,96 & 1,15 \\
\hline & & & 10 & 2,73 & 1,00 \\
\hline \multirow{7}{*}{ Conductual } & \multirow{7}{*}{2,90} & \multirow{7}{*}{1,33} & 5 & 3,06 & 1,25 \\
\hline & & & 6 & 2,64 & 1,12 \\
\hline & & & 7 & 2,04 & 1,05 \\
\hline & & & 8 & 2,14 & 1,09 \\
\hline & & & 12 & 3,99 & 1,01 \\
\hline & & & 14 & 2,40 & 1,05 \\
\hline & & & 18 & 4,07 & 1,02 \\
\hline \multirow{6}{*}{ Afectivo } & \multirow{6}{*}{2,89} & \multirow{6}{*}{1,14} & 9 & 3,23 & 0,98 \\
\hline & & & 11 & 2,71 & 1,12 \\
\hline & & & 13 & 3,07 & 1,20 \\
\hline & & & 15 & 3,19 & 1,07 \\
\hline & & & 16 & 2,55 & 1,19 \\
\hline & & & 17 & 2,58 & 1,10 \\
\hline
\end{tabular}

Fuente: elaboración propia.

Se puede señalar que el componente que presenta una mayor relativa actitud positiva fue el cognitivo (con el mayor promedio de 3,27 y con la menor desviación estándar de $1,10)$. Esto se sugiere que los estudiantes les otorgan importancia a las matemáticas y al conocimiento de la disciplina en su formación profesional. Tanto el componente conductual como el afectivo muestran el mismo nivel de importancia por los participantes, otorgando relevancia a las matemáticas en su formación $y$, al parecer, tienen agrado por la materia.
Con relación a las puntuaciones de los estudiantes por ítem, hemos seleccionado tres por cuestión de espacio, uno por cada componente, que presentan mayor media y menor desviación estándar, lo que sugiere una actitud con carga positiva.

En la Tabla 5 se presentan los porcentajes de las respuestas dadas por los estudiantes en el ítem 1, inscrito en el componente cognitivo, que busca indagar la actitud del futuro profesor cuando realiza una actividad matemática para la Educación Especial.

Tabla 5. Porcentajes de las respuestas de los estudiantes al ítem 1

\begin{tabular}{lccccc}
\multirow{2}{*}{ Semestre } & \multicolumn{4}{l}{ Ítem 1. Cuando me enfrento a una actividad matemática para la educación especial me siento capaz de resolverla. } \\
\cline { 2 - 6 } & $\begin{array}{l}\text { Totalmente en desacuerdo } \\
\text { Segundo }\end{array}$ & En desacuerdo & $\begin{array}{c}\text { Ni de acuerdo } \\
\text { ni en desacuerdo }\end{array}$ & De acuerdo & Muy de acuerdo \\
\hline Cuarto & 0,0 & 2,0 & 26,0 & 64,0 & 8,0 \\
\hline Sexto & 5,1 & 5,1 & 27,1 & 40,7 & 22,0 \\
\hline Octavo & 0,0 & 0,0 & 10,6 & 57,4 & 31,9 \\
\hline
\end{tabular}

Fuente: elaboración propia. 
Como se puede observar, el mayor porcentaje de estudiantes por semestre señala estar 'de acuerdo' en el ítem 1 (64 \%, 40,7 \%, 57,4 \% y 36,4 \% en segundo, cuarto, sexto y octavo semestre, respectivamente); esto sugiere que la mayoría de estudiantes de la Licenciatura en Educación Especial se consideran capaces de resolver actividades matemáticas.

En la Tabla 6 se muestran los porcentajes de las respuestas dadas por los estudiantes en el ítem 18, inscrito en el componente conductual, referente a la actitud del futuro profesor de Educación Especial sobre la aplicabilidad de las matemáticas en su cotidianidad.

Tabla 6. Porcentajes de las respuestas de los estudiantes al ítem 18

\begin{tabular}{|c|c|c|c|c|c|}
\hline \multirow[b]{2}{*}{ Semestre } & \multicolumn{5}{|c|}{ Ítem 18. Las matemáticas las aplico en mi vida cotidiana. } \\
\hline & $\begin{array}{l}\text { Totalmente en } \\
\text { desacuerdo }\end{array}$ & En desacuerdo & $\begin{array}{l}\mathrm{Ni} \text { de acuerdo } \\
\text { ni en desacuerdo }\end{array}$ & De acuerdo & $\begin{array}{l}\text { Muy de } \\
\text { acuerdo }\end{array}$ \\
\hline Segundo & 0,0 & 8,0 & 18,0 & $\underline{40,0}$ & 34,0 \\
\hline Cuarto & 6,8 & 5,1 & 23,7 & $\underline{39,0}$ & 25,4 \\
\hline Sexto & 0,0 & 0,0 & 17,0 & 38,3 & $\underline{44,7}$ \\
\hline Octavo & 6,8 & 2,3 & 0,0 & 25,0 & $\underline{65,9}$ \\
\hline
\end{tabular}

Fuente: elaboración propia.

Algo que se infiere respecto al ítem 18, en correspondencia con el mayor porcentaje del valor de la Escala de Likert obtenido por los estudiantes, atribuyendo importancia a la opción 'muy de acuerdo' (44,7 \% y 65,9 \% en sexto y octavo semestre, respectivamente), y 'de acuerdo' (40\% y $39 \%$ en segundo y cuarto semestre, respectivamente respectivamente), conjeturamos que, en general, los futuros profesores identifican que las matemáticas las aplican en su vida cotidiana. En particular, se observa un mayor porcentaje en los participantes de octavo semestre, posiblemente porque ya habían cursado la unidad de aprendizaje "adquisición, alteraciones y estrategias de atención en las matemáticas" y estaban en prácticas en escenarios reales (etapa de formación Práctica profesional).

En la Tabla 7 se presentan los porcentajes de las respuestas dadas por los estudiantes en el ítem 9, inscrito en el componente afectivo, referente a la tranquilidad del futuro profesor de Educación Especial cuando realiza actividades que implican matemáticas.

Tabla 7. Porcentajes de las respuestas de los estudiantes al ítem 9

\begin{tabular}{|c|c|c|c|c|c|}
\hline \multirow{2}{*}{ Semestre } & \multicolumn{5}{|c|}{$\begin{array}{l}\text { Ítem 9. Me siento tranquilo cuando tengo que trabajar en actividades que impliquen las } \\
\text { matemáticas. }\end{array}$} \\
\hline & $\begin{array}{l}\text { Totalmente en } \\
\text { desacuerdo }\end{array}$ & En desacuerdo & $\begin{array}{l}\mathrm{Ni} \text { de acuerdo } \\
\text { ni en desacuerdo }\end{array}$ & De acuerdo & Muy de acuerdo \\
\hline Segundo & 6,0 & 20,0 & 42,0 & 32,0 & 0,0 \\
\hline Cuarto & 1,7 & 27,1 & 39,0 & 25,4 & 6,8 \\
\hline Sexto & 2,1 & 6,4 & 36,2 & 46,8 & 8,5 \\
\hline Octavo & 6,8 & 15,9 & 31,8 & 25,0 & 20,5 \\
\hline
\end{tabular}

Fuente: elaboración propia. 
Al observar el mayor porcentaje del valor de la Escala de Likert obtenido por semestre, el $46,8 \%$ de los estudiantes de sexto semestre señala estar 'de acuerdo'; por otra parte, los estudiantes de segundo, cuarto y octavo semestre (42\%, $39 \%$ y 31,8\% respectivamente) señalan una postura 'neutra' en relación con su actitud afectiva hacia trabajar con actividades que impliquen el uso de matemáticas, esto al indicar 'ni de acuerdo ni en desacuerdo' en las puntuaciones dadas en la escala de Likert.

En la Tabla 8 se presenta un comparativo de las medias, y las desviaciones estándar, de las puntuaciones dadas por los futuros profesores de Educación Especial a cada uno de los ítems de los componentes de la actitud por semestre.

Tabla 8. Medias y desviaciones estándar de las puntuaciones por componentes en la actitud hacia las matemáticas por semestre

\begin{tabular}{|c|c|c|c|c|c|c|}
\hline \multirow{2}{*}{ Semestre } & \multicolumn{2}{|c|}{ Cognitivo } & \multicolumn{2}{|c|}{ Conductual } & \multicolumn{2}{|c|}{ Afectivo } \\
\hline & Media & Desviación Estándar & Media & Desviación Estándar & Media & Desviación Estándar \\
\hline Segundo & 3,13 & 1,02 & 3,09 & 1,13 & 2,98 & 1,14 \\
\hline Cuarto & 3,06 & 1,18 & 2,83 & 1,26 & 2,88 & 1,17 \\
\hline Sexto & 3,52 & 0,99 & 2,93 & 1,43 & 2,83 & 1,06 \\
\hline Octavo & 3,44 & 1,12 & 2,76 & 1,51 & 2,84 & 1,20 \\
\hline
\end{tabular}

Fuente: elaboración propia.

Se puede apreciar que, en promedio, los estudiantes de sexto semestre exhiben una mayor actitud positiva en el componente cognitivo, mientras que los de segundo semestre muestran una actitud positiva en el aspecto conductual y afectivo hacia las matemáticas.

Un punto a señalar es el hecho de que los estudiantes del octavo semestre tengan una actitud negativa en el componente conductual con relación a la asignatura; ya que, como lo sugiere Casis et ál. (2017), una actitud negativa pudiera influir en la futura práctica profesional de los estudiantes y limitar la motivación, la perseverancia y el autoconcepto hacia las matemáticas (Ruiz et ál., 2013), pues estos en ese nivel se encuentran realizando sus prácticas en escenarios reales.

En síntesis, según las componentes de la actitud consideradas en líneas arriba y los mayores promedios de la escala en cada ítem, en la Tabla 9 se muestran las características de la actitud positiva hacia las matemáticas de futuros profesores de Educación Especial. 
Tabla 9. Caracterización de las actitudes de los futuros profesores de Educación Especial

\begin{tabular}{ll} 
Componente & Característica \\
Cognitivo & $\begin{array}{l}\text { a) Los estudiantes se sientes capaces de resolver situaciones matemáticas } \\
\text { brofesional } \\
\text { c) Los estudiantes consideran importante poseer un alto dominio de las matemáticas } \\
\text { para su desarrollo profesional }\end{array}$ \\
\hline & $\begin{array}{l}\text { a) Los estudiantes consideran indispensable la buena disposición del profesor al } \\
\text { impartir matemáticas }\end{array}$ \\
b) Los estudiantes les otorgan relevancia a las matemáticas al considerarlas que se \\
aplican en la vida cotidiana
\end{tabular}

Fuente: elaboración propia.

\section{Conclusiones}

Del análisis anterior podemos concluir que la mayor proporción de los estudiantes de los cuatro semestres presentan una predisposición positiva hacia la asignatura de Matemáticas y su contenido, se sienten capaces de resolver actividades matemáticas para la Educación Especial y, en general, la consideran relevante en su formación profesional.

También se puede señalar que los estudiantes de sexto y octavo semestre tienen una acepción distinta de las matemáticas, lo que sugiere otorgarle importancia al contenido, su aplicación en la vida cotidiana y en su preparación profesional; al parecer, esto debido a que ya cursaron la unidad de aprendizaje correspondiente y por tener prácticas en escenarios reales. En tanto los estudiantes del segundo semestre quizá tengan experiencias previas positivas con las matemáticas (Ruiz et ál., 2013).

Respecto al componente afectivo, se identificó que los estudiantes de sexto semestre presentan un gusto por las matemáticas y se sienten tranquilos al trabajar en actividades que las involucran. En los demás semestres (segundo, cuarto y octavo), la mayoría centró su preferencia en neutro. Lo curioso es que a pesar de que los alumnos de octavo semestre ya habían cursado la unidad de aprendizaje relacionada a las matemáticas, no se mostró una mayor actitud positiva en el componente afectivo. Por lo que, si las experiencias se repiten y su resultado lleva a emociones negativas, es probable que se transforme en una actitud negativa (Espinosa y Oliver, 2012; Martínez-Sierra, 2015; Sánchez et ál., 2011).

A partir de lo señalado por Espittia (201 1), sobre la relación entre la motivación del profesor respecto al proceso de enseñanza y aprendizaje de las matemáticas con el desencadenamiento de actitudes positivas por parte del aprendizaje de los estudiantes, esta investigación pretendió documentar las actitudes posi- 
tivas que pudieran desencadenar una mejor comprensión de las matemáticas por parte de los futuros profesores de Educación Especial. En ese sentido, provocar desde la formación inicial actitudes positivas provocaría un mejor agrado hacia las matemáticas por parte de los estudiantes y, por tanto, un mejor conocimiento del contenido (Bravo, 2014).

Por lo tanto, conjeturamos que después de cursar la unidad de aprendizaje "adquisición, alteraciones y estrategias de atención en las matemáticas", los futuros profesores identifican que es necesario tener un alto dominio en la materia para poder asesorar a los padres de familia o alumnos frente a las dificultades de aprendizaje de esta área del conocimiento.

\section{Referencias}

Bravo, M. (2014). Actitudes hacia las matemáticas y rendimiento académico en estudiantes de secundaria: un enfoque cuantitativo. [Tesis de licenciatura, Benemérita Universidad Autónoma de Puebla]. Repositorio institucional BUAP. https://hdl. handle.net/20.500.12371/5331

Casis, M., Rico N. y Castro, E. (2017). Motivación, autoconfianza y ansiedad como descriptores de la actitud hacia las matemáticas de los futuros profesores de educación básica de chile. PNA: Revista de Investigación en Didáctica de la Matemática, 17 (3), 181-203.

Di Martino, P. (2016). Attitude. En G. Kaiser (Ed.). Attitudes, Beliefs, Motivation and Identity in Mathematics Education. An Overview of the Field and Future Directions (pp. 2-7). Springer.

Espinosa, C. y Oliver, E. (2012). Evaluación sobre los perfiles de ingreso de los alumnos de los posgrados de administración: actitudes y experiencias hacia las matemáticas. Revista de Currículum y Formación de Profesorado, 14(1), 361-377.

Espittia, S. (201 1). Actitudes hacia el aprendizaje de la matemática, habilidades lógico matemáticas y los intereses para su enseñanza, en estudiantes de educación, especialidad primaria de la UNMSM. [Tesis de maestría, Universidad Nacional Mayor de San Marcos]. Repositorio institucional UNMSM https://hdl. handle.net/20.500.12672/1668

Fennema, E. y Sherman, J. A. (1976). Fennema-Sherman mathematics attitude scale. Instruments designed to measure attitudes toward the learning of mathematics by male and female. JSAS Catalog of Selected Documents of Psychology, VI(31), 1-31.

Flores, J. y García-Cedillo, I. (2016). Apoyos que reciben estudiantes de secundaria con discapacidad en escuelas regulares: $\dot{c}$ corresponden a lo que dicen las leyes? . Revista Educación, 40(2), 1-20.

García-Cedillo, I. (2018). La educación inclusiva en la Reforma Educativa de México. Revista de Educación Inclusiva, 17 (2), 49-62.

García-Cedillo, I. y Romero, S. (2016). Avances de la integración educativa/educación inclusiva y la formación docente para la inclusión en México. CeneJus-UAsLP.

García-Cedillo, I. y Romero, S. (2019). Influencia de la Declaración de Salamanca sobre la Atención a la Diversidad en México y Situación Actual. Revista Latinoamericana de Educación Inclusiva, 13(2), 123-138.

García, M. y Farfán, R. (2018). El saber matemático en la formación de actitudes. En C. Dolores, G. Martínez, M. García, J. Juárez y Ramírez (Eds.)., Investigaciones en dominio afectivo en matemática educativa (191-210). Ediciones Eón. 
García, M. y Juárez, J. (2011). Revisión del constructo actitud en Educación Matemática: 1959-1979. Unión: Revista Iberoamericana de Educación Matemática, 26, $117-125$.

García, M. y Pascual, M. (2018). De la congoja a la satisfacción: el conocimiento emocional del profesor de matemáticas. IE Revista de investigación educativa de la REDIECH, 8(15), 133-148. https://doi.org/10.33010/ie_rie_rediech.v8i15.68

Gil, N., Blanco, L. y Guerrero, E. (2005). El dominio afectivo en el aprendizaje de las matemáticas. Una revisión de sus descriptores básicos. Unión: Revista Iberoamericana de Educación Matemática, 2, 15-32.

Gómez-Chacón, I. (2009). Actitudes matemáticas: propuestas para la transición del bachillerato a la universidad. Revista Educación Matemática, 21 (3), 5-32.

Guajardo, E. (2010). La desprofesionalización docente en educación especial. Revista Latinoamericana de Educación Inclusiva, 4(1), 105-126.

Hannula, M., Evans, J., Philippou, G. y Zan, R. (2004). Affect mathematics education-exploring theoretical frameworks. En M. Høines Y A. Fuglestad (Eds.). Proceedings of the 28th PME International Conference, 107-136.

Hegarty, S. (2008). Investigación sobre educación especial en Europa. Revista Iberoamericana sobre Calidad, Eficacia y Cambio en Educación, 2(6), 119-199.

López-Mojica, J. M. y Aké, L. P. (2015). Formación matemática del docente de educación especial. En P. Scott y A. Ruíz (eds.). Educación Matemática en las Américas: 2015. Volumen 14: Necesidades Especiales (94-101). CIAEM.

Ma, X. y Kishor, N. (1997). Assessing the Relationship between Attitude toward Mathematics and Achievement in Mathematics: A Meta-Analysis. Journal for Research in Mathematics Education, 28, 26-47.

Martínez-Artero, R. y Nortes C. (2013). Actitud hacia las Matemáticas en futuros docentes de primaria y de secundaria. Edetania, 44, 47-76.

Martínez-Padrón, O. (2008). Actitudes hacia las matemáticas. Sapiens: Revista Universitaria de Investigación, 9(1), 237-256.

Martínez-Sierra, G. (2015). Students emotional experiences in high school mathematics classroom. En K. Krainer, N. Vondrov'a (Eds.) CERME 9 - Ninth Congress of the European Society for Research in Mathematics Education (1181-1187). Charles University.

Monje, C. (201 1). Metodología de la investigación cuantitativa y cualitativa. Una guía didáctica. Universidad Surcolombiana.

Palella, S. y Martins, F. (2012). Metodología de la investigación cuantitativa. Fedupel.

Perrenoud, P. (2010). Diez nuevas competencias para enseñar. Grao.

Ramírez, J. C. y López-Mojica, J. M. (2015). Un análisis curricular de la formación de profesionistas de la educación especial en matemáticas. En J. López y J. Cuevas 
(eds.), Educación especial y matemática educativa. Una aproximación desde la formación docente y procesos de enseñanza (53-71). Cenejus.

Ramírez, J. C., López-Mojica, J. M. y Aké, L. P. (2018). Importancia de las matemáticas en la formación inicial de profesionistas de la educación especial. Atenas: Revista Científico Pedagógica, 43(3), 100-114.

Ramírez, J. C., Verdúzco, M. y García, A. (2018). Validez y confiabilidad de una escala para medir actitudes hacia las matemáticas en educación especial. En C. Dolores, G. Martínez, M. García, J. Juárez, Ramírez (Eds.)., Investigaciones en dominio afectivo en matemática educativa (243-259). Guerrero: Ediciones Eón.

Romero, S. y García-Cedillo, I. (2013). Educación especial en México. Desafíos de la educación inclusiva. Revista Latinoamericana de Educación Inclusiva, 7(2), 77-91.

Roesken, B., Hannula, M. y Pehkonen, E. (201 1). Dimensions of students' views of themselves as learners of mathematics. ZDM Mathematics Education 43, 497-506.

Ruiz, J., García, J. y Sarasua, J. (2013). Perspectivas de los alumnos de grado de educación primaria sobre las matemáticas y su enseñanza. Revista Números, 82, 5-15.

Sánchez, J., Segovia, I. y Miñán, A. (2011). Exploración de la ansiedad hacia las matemáticas en los futuros maestros de educación primaria. Revista de Currículum y Formación de Profesorado, 15(3), 297-312.

Secretaría de Educación Pública, SEP. (2004). Licenciatura en educación especial. Programa para la transformación y el fortalecimiento académicos de las escuelas normales. Plan de estudios. SEP.
Secretaría de Educación Pública, SEP. (2016). Aprendizajes clave para la educación integral. Plan y programas de estudios para la educación básica. SEP.

Organización de las Naciones Unidas para la Educación, Unesco. (1994). World Conference on Special Needs Education: Access and Quality, Salamanca, Spain. The Salamanca Statement and Framework for Action on Special needs Education. Paris.

Universidad de Colima. (2011). Curriculum Integrado Centrado en el Aprendizaje. Licenciatura en Educación Especial. UdC.

Valdés, A. J. y Monereo, C. (2012). Desafíos a la formación del docente inclusivo: la identidad profesional y su relación con los incidentes críticos. Revista Latinoamericana de Educación Inclusiva, 6(2), 193-208.

Zapata, M. (2019). Más allá de la profesionalización: los quehaceres de la educación. Tecné, Episteme y Didaxis: TED, (46), 139-156. https://doi.org/10.17227/ted.num46-10544

\section{Como citar este artículo:}

López-Mojica, J. M., García-García, J. I., Ramírez, J. C., y Arredondo, E. H. (2021). Exploración de las actitudes hacía las matemáticas de futuros profesores de educación especial. Tecné, Episteme y Didaxis: TED, (50), 95-112. https://doi.org/10.17227/ted.num5014210. 